\title{
Experimental and Numerical Investigation of Centrifugal Vortex Pump Operating Benefits for Energy Efficient Systems
}

\author{
Tihomir MIHALIĆ*, Živko KONDIĆ, Srđan MEDIĆ, Veljko KONDIĆ
}

\begin{abstract}
One of the ways to improve the performance of centrifugal pumps, proposed and experimentally validated by our research, is the method of creating so-called coherent structures, vortices and turbulence in the peripheral area of the centrifugal stage by adding a vortex rim to the back side of centrifugal rotor. A new construction of the pump, so called centrifugal vortex pump (CV), combines the good sides of these two types of pumps: high head of the vortex pump and high efficiency of the centrifugal pump. In addition, centrifugal pumps deliver higher flow rates with lower pressures, vortex pumps deliver higher pressures but with lower flow rates. For centrifugal vortex pumps, the vortex blades are located at the rear of the centrifugal rotor. The outer diameter of the vortex rim (VR) is smaller than the outer diameter of the centrifugal rotor (CR). The vortex rim induces vorticity to a portion of the flow that has passed through the centrifugal rotor. This vortexed flow is then reunited with the rest of the flow that has not been pulled down by vortex rotor. The vortex energy of that additional stream transfers some of its kinetic energy to the main stream. This added kinetic energy is converted to a pressure that accumulates the pressure exerted by the centrifugal rotor, and thus the vortex rim improves the overall pump performance. An intense process of gas dispersion occurs in the vortex region, which increases the stability of the pump when pumping a mixture of liquids and gases. The process of energy conversion in a centrifugal vortex pump, i.e., flow visualisation of mixing streams from a centrifugal rotor and a vortex rotor, and the quantization and superpositions have been shown in this paper.
\end{abstract}

Keywords: coherent structures; energy of vortices; energy transformation; unsteady flow; vortexed flow; vortex rim

\section{INTRODUCTION}

The kinetic energy of coherent structures and vortices, which is created by the vortex rim, is added to the flow of fluid flowing out of the centrifugal rotor channel. In this way the vortexed flow increases the total energy of the fluid before entering the stator. Furthermore, longitudinal vortices, which occur along the edges of the VR due to changes in the kinetic energy of the vortex, and radial vortices, which split off from it, transmit additional energy to the main fluid flow. The intensity of the vortex process and the corresponding pressure increase depend on the flow, and with the given flow - on the size of the axial component of the absolute velocity when the liquid flows from the centrifugal rotor into the diffusor. The lower the flow rate and the smaller the axial velocity component, the greater number of channels of the vortex rim (VR) is involved in changing the momentum of fluid, which is at a given time entrapped in those channels, Fig. 1, [1-5].

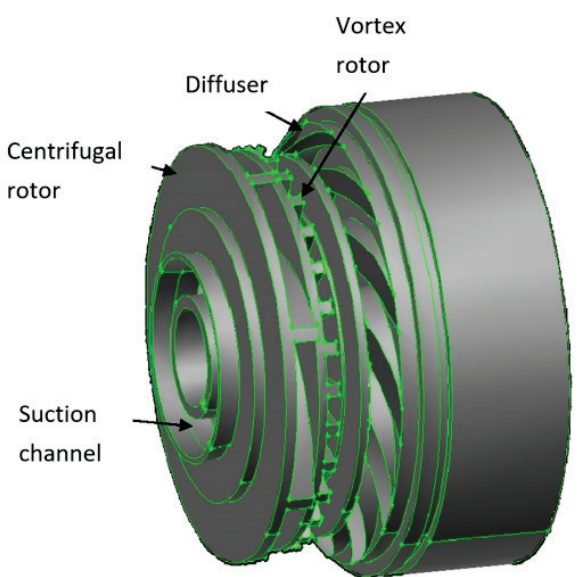

Figure 1 Centrifugal vortex rotor [5]

Zhejiang Sci-Tech University, [6], has performed experimental studies on vortex centrifugal pump, where the centrifugal and vortex rotor are on the same shaft, but separated Fig. 2. They give a useful experimental data in the form of $Q-H$ and $Q-\eta$ diagrams, for the case when the working fluid was water and the mixture of water and gas. From their results it can be seen that this centrifugal vortex pump has improved supply and the flow cut off even with the $15 \%$ higher gas content in the liquid, compared to the centrifugal pump.

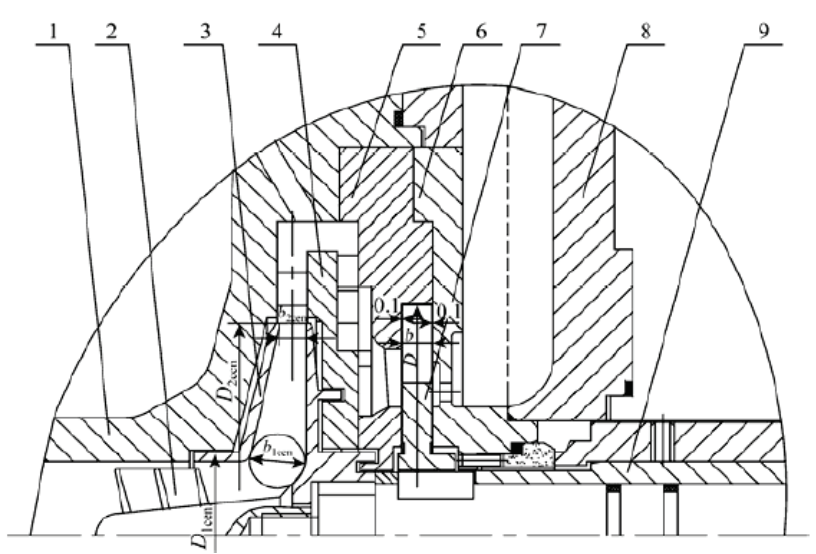

Figure 2 Centrifugal vortex pump stage with separated centrifugal and vortex rotor; 1-inlet casing; 2-inducer; 3-centrifugal impeller; 4-interstage diffuser; 5suction; 6-discharge;7-vortex impeller; 8-outlet casing; 9-shaft sleeve, [6]

In order to better understand the energy conversion, which is the result of the interaction of the flow from the centrifugal rotor channels with eddies formed in the vortex rim and their interaction with the diffusor, an analysis of the flow structures was carried out. In this paper experimental and computational methodology is presented.

\section{MODELS OF TESTED PUMP STAGES \\ 2.1 Experimental Models}

The experimental assembly, Fig. 3, started with a valve at the entrance, followed by a $1 \mathrm{~m}$ plexiglas tube, then venturi tube, Fig. 4 , then $1 \mathrm{~m}$ plexiglas tube with measuring points for suction pressure, then the metering section containing various pump stages, Fig. 5 , followed by a $1 \mathrm{~m}$ 
plexiglas tube with the measuring points for discharge pressure and the outlet valve. Described measuring assembly was built by governing ISO standards.

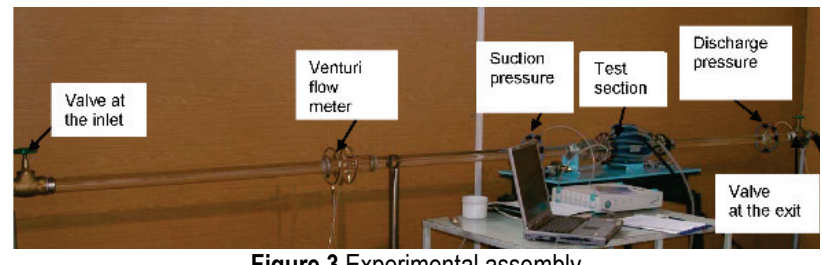

Figure 3 Experimental assembly

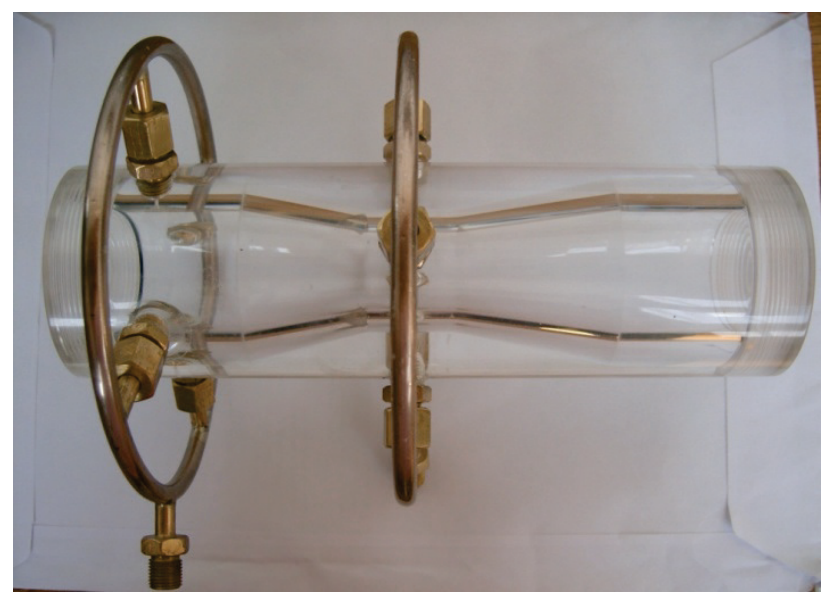

Figure 4 Venturi tube [5]

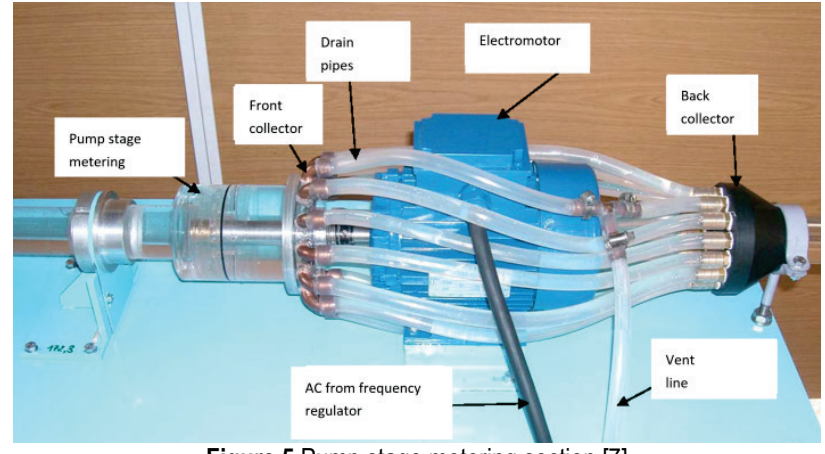

Figure 5 Pump stage metering section [7]

\subsection{Error Estimate of the Used Numerical Method and Models}

In order for any result obtained by CFD (Computational Fluid Dynamics) to be considered as a valid result, it is necessary to know how confident they are. The purpose of uncertainty estimation is to limit the simulation errors, that is, to keep them under control. It is necessary to be able to discern the effects on uncertainty that arise from numerical errors and those that arise from model errors.

The components of CFD uncertainty are: 1 . Input data uncertainty, 2. Model uncertainty, and 3. Numerical uncertainty. Numerical uncertainty is the only one that cannot be eliminated, and its sources lie in spatial discretization errors due to insufficient geometric mesh resolutions and in time discretization errors due to large time step, in incomplete convergence of an iterative solver, and in computer program errors, [8].

In order to determine spatial and temporal discretization errors, several refinements of the control volumes $(\mathrm{CV})$ grids and time steps were performed, [9].
In this paper, a method for estimating the spatial discretization error was used [10], which consisted of the following steps:

Step 1: A representative $C V$ size $\Delta$ was defined from:

$\Delta=\left[\frac{1}{N} \sum_{i=1}^{N}\left(\Delta V_{i}\right)\right]^{1 / 3}$

where: $\Delta V_{i}$ is the volume of the $i^{\text {th }} C V$ and $N$ is the total number of $C V$ in the $3 \mathrm{D}$ domain.

Step 2: Three significantly different $C V$ meshes were generated and simulations were performed to determine the values of key variables relevant to the simulation result. The mesh refinement factor $\left(r=\Delta_{\text {coarse }} / \Delta_{\text {finer }}\right)$ was greater than 1.3; this was shown to be the best.

Step 3: As $\Delta_{1}<\Delta_{2}<\Delta_{3}$ and $r_{21}=\Delta_{2} / \Delta_{1}, r_{32}=\Delta_{3} / \Delta_{2}$; the "apparent" order of accuracy of method $p$ is calculated using the equations:

$$
\begin{aligned}
& p=\frac{1}{\ln \left(r_{21}\right)}|\ln | \frac{\varepsilon_{32}}{\varepsilon_{21}}|+q(p)| \\
& q(p)=\ln \left(\frac{r_{21}^{p}-s}{r_{32}^{p}-s}\right) \\
& s=1 \cdot \operatorname{sgn}\left(\frac{\varepsilon_{32}}{\varepsilon_{21}}\right)
\end{aligned}
$$

where: $\Phi_{i}$ is the numerical result of chosen physical variable on different grids $(i=1,2,3), \varepsilon_{32}=\Phi_{3}-\Phi_{2}$ and $\varepsilon_{21}=\Phi_{2}-\Phi_{1}$.

Step 4: Extrapolated value of chosen physical variable $\Phi$ was calculated from:

$$
\Phi_{\mathrm{ext}}^{21}=\frac{\left(r_{21}^{p} \Phi_{1}-\Phi_{2}\right)}{\left(r_{21}^{p}-1\right)}
$$

Step 5: Estimated error was calculated from:

$$
e r_{\mathrm{a}}^{21}=\left|\frac{\Phi_{1}-\Phi_{2}}{\Phi_{1}}\right|
$$

Extrapolated relative error was calculated from:

$$
e r_{\mathrm{ext}}^{21}=\left|\frac{\Phi_{\mathrm{ext}}^{12}-\Phi_{1}}{\Phi_{\mathrm{ext}}^{12}}\right|
$$

$G C I_{\text {fine }}^{21}$ (Grid Convergence Index) was then calculated from:

$$
G C I_{\text {fine }}^{21}=\frac{1.25 e r_{\mathrm{a}}^{21}}{r_{21}^{p}-1}
$$


Factor 1.25 in Eq. (8) is actually a factor of safety that was increased to 3 in this research to be on more conservative side of the solution. If the value of this factor were 1 , the results of the numerical simulations would fall into that range with $50 \%$ certainty. Therefore, factor 3 corresponds to a $99.73 \%$ (normal distribution) certainty that the actual CFD result will be in the range of $\Phi_{\text {ext }} \pm G C I$.

\section{RESEARCH RESULTS}

\subsection{Numerical Estimate of the Energy Contribution of the Vortex Rim Mounted on the Backside of Centrifugal Rotor}

The contribution of the vortex rim to the total head and to the corresponding pressure increase depended on the flow. At the specific flow, this contribution depended on the magnitude of the axial component of the absolute fluid velocity flowing from the centrifugal rotor to the diffuser ducts. The smaller the flow rate and the smaller the axial velocity component, the greater the number of VR blades participated in changing the momentum of fluid particles. Fig. 6, clearly shows that the VR in our centrifugal vortex pump began to increase the flow rate from $105 \mathrm{~m}^{3} /$ day $(1.22 \mathrm{l} / \mathrm{s})$ to zero. This is very beneficial because the pump in that operating range was more attenuated; it needed to overcome larger resistance at the outlet. As this flow resistance became larger, VR rim helped more thus its energy contribution was increasing, [7].

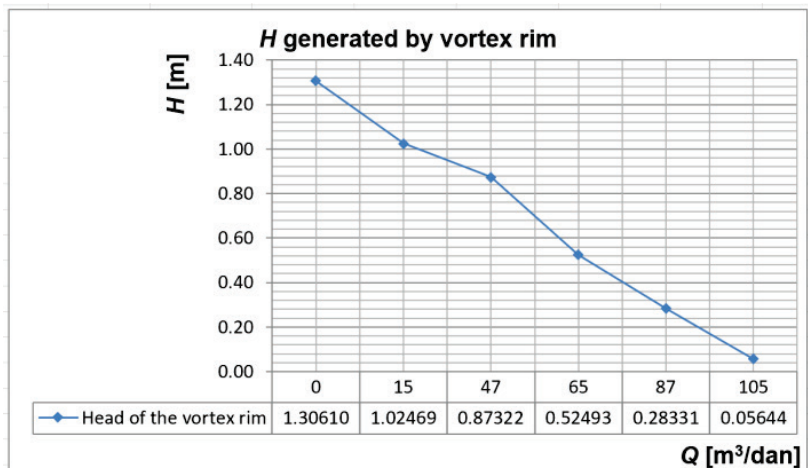

Figure 6 Contribution of energy flows in the vortex rim to increase total $H, n=$ $2910 \mathrm{~min}^{-1}$

\subsection{Analysis of the Pressure Filed in Centrifugal Vortex and Centrifugal Pump Stage}

For the pressure filed analysis, rotors were sectioned by the perpendicular plane to the rotation axis, exactly at the half width of the centrifugal blades, [11].
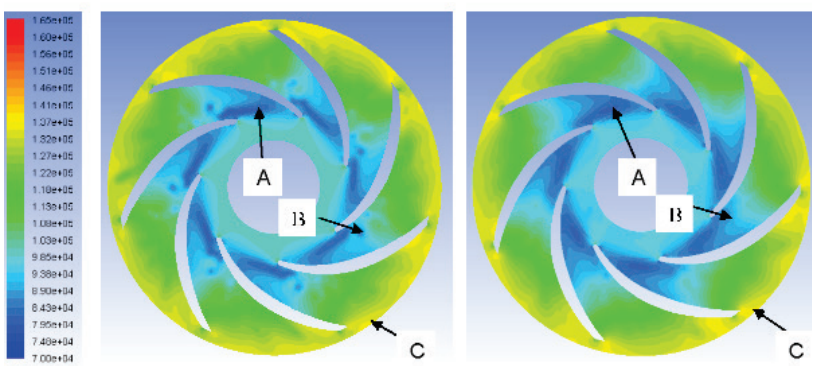

Figure 7 Pressure filed in centrifugal vortex (left) and centrifugal (right) pump stage; $k_{\mathrm{L}}=0$
Comparing the suction areas A in Fig. 7 it can be concluded that the centrifugal pump creates a much wider suction area with the same head and supply.

Area of the lower pressure at the centrifugal vortex pump collapses more quickly by the vortices shredding, area B in Fig. 7, while this transition in the centrifugal pump is more continuous. The only logical conclusion is that the VR affects the suction area by accelerating this transition.

Attenuating flow, $k_{\mathrm{L}}=5$, the pressure fields and the form of suction area, for both pumps becomes similar, Fig. 8. It can be seen that the point of the lowest pressure (highest underpressure), in both pumps, is located just below leading edge of the convex side of the rotor blades (point A).
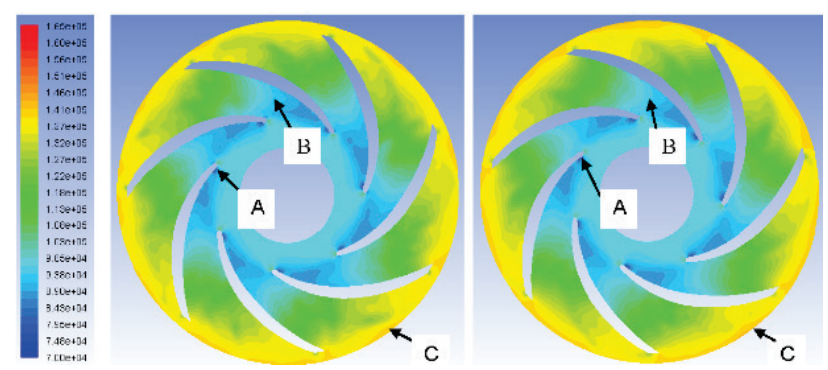

Figure 8 Pressure filed in centrifugal vortex (left) and centrifugal (right) pump stage; $k_{\mathrm{L}}=5$

This suction pressure (point A) is lower in the centrifugal pump. Unlike the previous working regime of the centrifugal vortex pump, here the pressure increases gradually over the isobars which are perpendicular to the convex side of the rotor blade (point B).

Fig. 9 shows the pressure fields in the pumps when $k_{\mathrm{L}}$ $=60$. It can be seen that the suction area at both pumps quickly collapses.
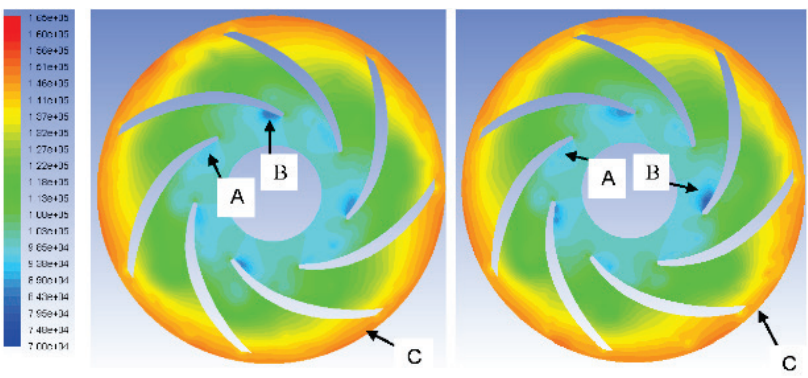

Figure 9 Pressure filed in centrifugal vortex (left) and centrifugal (right) pump stage; $k_{\mathrm{L}}=60$
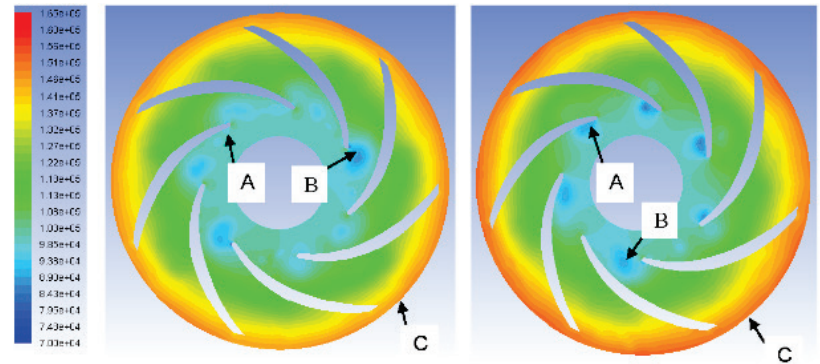

Figure 10 Pressure field in centrifugal vortex (left) and centrifugal (right) pump stage; $k_{\mathrm{L}}=300$

This suction area (point A and point B) is more concentrated and at the centrifugal vortex pump can be on the convex side of the blades, but also away from them, 
somewhere in the flow. Integrating static and dynamic pressure at the perimeter of the rotor in this regime gives that centrifugal pump realizes higher static pressure but lower dynamic pressure than the centrifugal vortex pump.

Fig. 10 shows the pressure field in pumps at final attenuation of the flow (fully closed valve at the pump discharge) $k_{\mathrm{L}}=300$. The suction area at both pumps quickly transits to higher pressure. The lowest pressure point (point $A$ and point $B$ ), in the centrifugal vortex pump, is in front of the leading edge of the blades and away from the blades in the fluid.

This lowest pressure point, in the centrifugal pump, is still on the convex side of the blades.

From the analysis of the lowest pressure in the pumps working at different regimes a diagram shown in Fig. 11, can be derived.

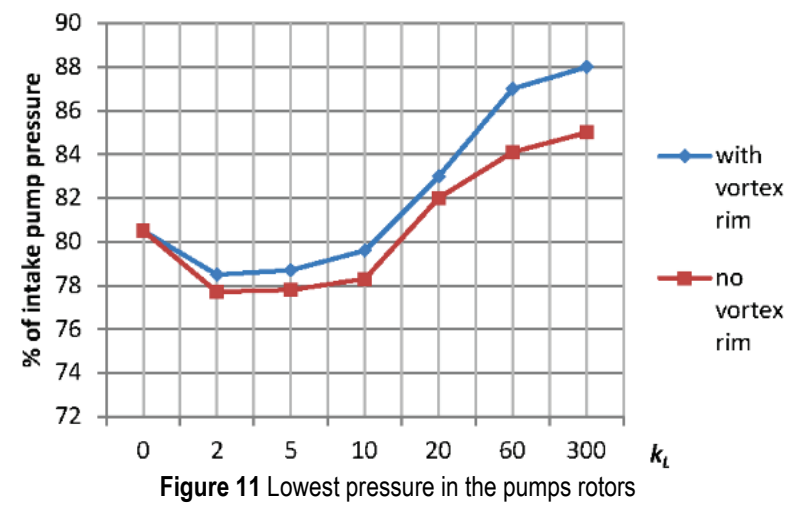

Centrifugal vortex pump generates higher suction pressure (smaller underpressure) while giving higher head and supply. The biggest difference of these suction pressures occurs at the end of the working regime at the full flow attenuation. From the loss coefficient $k_{\mathrm{L}}=20$ towards $k_{\mathrm{L}}=300$, centrifugal rotor of the centrifugal vortex pump starts to lag in its work capacity and the VR starts to compensate, allowing it to establish smaller underpressure.

How much lower can suction pressure be, at the centrifugal vortex pump, is determined by the vortex rim. The maximum static overpressure generated by the vortex rim at its outer diameter is the static overpressure which centrifugal rotor must generate at the same diameter because vortex rim is downstream centrifugal rotor.

At higher angular velocities, vortex rim creates more and more coherent structures so higher and higher dynamic pressure is achieved, producing lower and lower static overpressure. To preserve the balance, centrifugal rotor must follow these tendencies imposed by the vortex rim. For that, centrifugal rotor also needs to generate lower static overpressure at its outer diameter, and for this it needs a smaller underpressure at the pump intake.

Vortex rim acts as the regulator for the centrifuge rotor of the centrifugal vortex pump. This property is important for the stability of the pump cavitation.

\subsection{Streamlines Analysis in Centrifugal Vortex and Centrifugal Pump}

To analyse energy transfers from VR to fluid particles we were looking at the streamlines coloured by $\boldsymbol{Q}$ criterion defined by Eq. (9). $\boldsymbol{Q}$ criterion represents "lower pressure tubular formations", coherent structures by which most of the energy is transported from the VR to fluid particles. The pressure difference is mostly pronounced in the vortex nucleus so the "lower pressure tubular formations" actually represent the vortex nucleus.

$\boldsymbol{Q}=\frac{1}{2}\left(\|\boldsymbol{W}\|^{2}-\|\boldsymbol{S}\|^{2}\right)$

where: $\boldsymbol{Q}$ is the second invariant of the velocity gradient tensor; $\boldsymbol{W}$ - asymmetric part of the velocity gradient tensor; $\boldsymbol{S}$ - local value of deformation tensor.

From Fig. 12, it was concluded that the VR is a generator of a large number of coherent structures, as evidenced by the large number of yellow and red coloured streams opposite to the regular centrifugal rotor (CR). There were also many streamlines on the VR that formed a closed loop or had a spiral pattern, [12]. On the back side of CR streamlines were almost concentric circles, with very low values of $\boldsymbol{Q}$ criteria.
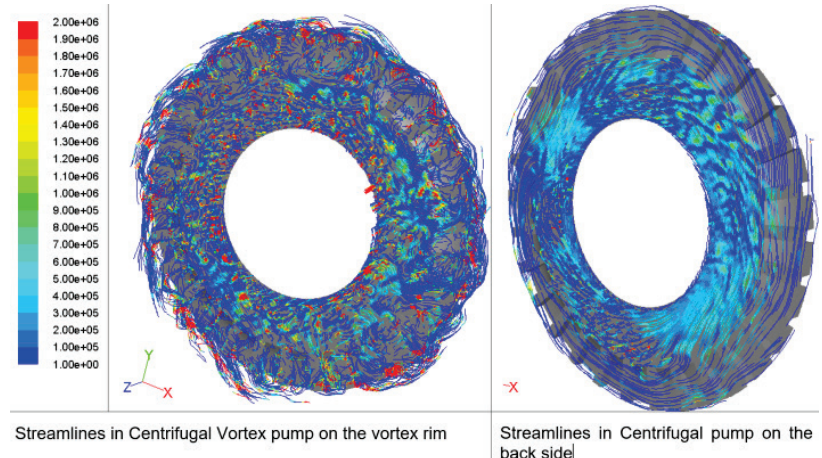

Figure 12 Streamlines colored by $\boldsymbol{Q}$ criterion, $k_{\mathrm{L}}=60, n=2910 \mathrm{~min}^{-1}$

Only by approaching the rotation axis of the centrifugal rotor $(\mathrm{CR})$ the values of the criterion increased and the streamlines lost concentricity and some vortexes were produced. By approaching the axis of rotation, the gap between the fixed walls of the stator, Fig. 1, is reduced, and this in fact represents the flow between two disks slightly apart from each other. Such flow describes Tesla's pump/turbine, [13].

From Fig. 12 a pattern of vortex formation was visualized, where the region of vortex nucleus formation (light blue) and the region without vortex nucleation formation (dark blue) appear alternately. This periodicity is a property of Taylor vortices [14]. This was the main difference spotted between fluid flow over VR and the back side of centrifugal rotor. On the VR coherent structures were formed while on the back side of CR Taylor vortices were formed.

Fig. 13, shows streamlines coloured by the mentioned criterion at maximum flow; pump stage is not attenuated. Observing the difference between Fig. 12 and Fig. 13, it can be concluded that, at high flows, VR took a much smaller fraction of the fluid from at the main flow leaving centrifugal rotor. Therefore, $\boldsymbol{Q}$ criterion showed that the VR generated Taylor antisymmetric vortex pairs from its axis of rotation to its blades, while it was generating coherent structures at smaller flow rates.

By analysing the flows at high and low flow rates of the CV and C pump, it was concluded that the VR produced two flow structures. It created turbulent flow but also coherent structures. At low flow rates, the formation of 
coherent structures was more dominant, while at higher flow rates, turbulent flow was more dominant. Turbulence increases the pressure drop by increasing the irreversibility of the process (increasing entropy), so at high flow rates his increased losses of the CV pump. In contrast, its contribution at low flow rates to increase pump head $H$ and contributing to the formation of coherent structures outweighs its impact on increasing irreversibility, [15].

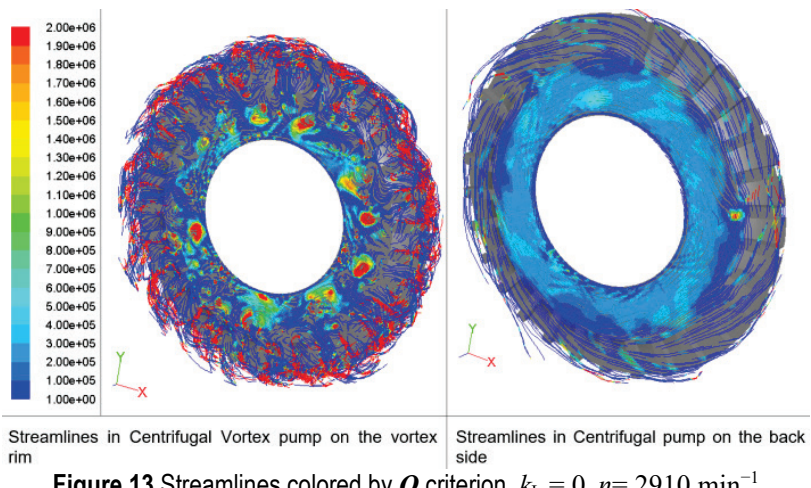

Figure 13 Streamlines colored by $\boldsymbol{O}$ criterion, $k_{\mathrm{L}}=0, n=2910 \mathrm{~min}^{-1}$

\section{CONCLUSION}

It was proven that vortex rim helps centrifugal rotor in two ways. In the first way, vortex rim captures fluid that did not enter the stator, because of the lack of energy, and gives it additional energy by accelerating it to a higher diameter. In the second way, it generates coherent structures which act like mediator in transferring energy from vortex rim to fluid particles.

In further research, it was shown that vortex rim regulates suction underpressure in the centrifugal rotor. This suction underpressure is smaller than in the similar centrifugal pump providing delay of cavitation and hence increasing working stability. CFD simulations of the cavitation have shown that at the same working regime (installation characteristic) centrifugal pump starts to cavitate at $20 \%$ lower angular velocity than centrifugal vortex pump. It has been shown that cavitation at the centrifugal vortex pump originates in the fluid not on blades.

It is assumed that the centrifugal vortex pump will have better performances than centrifugal pumps when working fluid is multiphase. This must be verified in a further research but the blades of the vortex rim should have positive effect if there are gas bubbles or even sludge in the working fluid.

It can be concluded that the centrifugal vortex pumps have some key operating benefits that can be decisive factor in their development and usage. Their benefits are manifested in extreme working conditions (low flow, high system resistance), so if head and cavitation characteristics are more important than energy consumption, it is reasonable to use centrifugal vortex instead plain centrifugal pump.

\section{REFERENCES}

[1] Mihajlovič, P. O., Jurevič, M. I., Borisovič, K. P., Isaakovič, R. A., \& Pavlovič, T. I. (2001). New rotary-vortex pumps for crude oil production in the complicated conditions. 1 . International conference of technical sciences, 25-37.

[2] Isaakovič, R. A. \& Vasiljevič, G. N. (2003). High-head, economical modifications of multistage centrifugal pump for oil production. 2. International conference of technical sciences, 125-140.

[3] Karakulov, S. T., Meljnikov, D. J., Pereljmman, M. O., \& Dengaev, A. V. (2005). Ways of increasing efficiency of oil exploitation from oil wells. 3. International conference of technical sciences, 78-87.

[4] Melzi, E. (2008). Vortex impeller for centrifugal fluiddynamic pumps. European Patent Application EP1 961965 A2.

[5] Mihalić, T., Guzović, Z., \& Predin, A. (2013). Performances and Flow Analysis in the Centrifugal Vortex Pump.Journal of fluids engineering, 1(011002-1), 135-142. https://doi.org/10.1115/1.4023198

[6] Zuchao, Z., Peng, X., Guofu, O., Baoling, C., \& Yi, L. (2008). Design and experimental analyses of small-flow high head centrifugal-vortex pump for gas-liquid two phase mixture. Chinese Journal of Chemical Engineering, 16, 528534. https://doi.org/10.1016/S1004-9541(08)60116-0

[7] Mihalić, T., Medić, S., \& Kondić, Ž. (2013). Improving Centrifugal Pump By Adding Vortex Rotor. Tehnički vjesnik, 20(2), 305-309.

[8] Freitas, C. J. (1999). The issue of numerical uncertainty, CSIRO, 29-34

[9] Roache, P. J. (1997). Quantification of uncertainity in computational fluid dynamics. Annual Rev. Fluid Mech, 123-160. https://doi.org/10.1146/annurev.fluid.29.1.123

[10] Mihalić, T., Guzović, Z., \& Predin, A. (2014). CFD flow analysis in the centrifugal vortex pump.International journal of numerical methods for heat \& fluid flow, 24(3), 545-562. https://doi.org/10.1108/HFF-05-2012-0124

[11] Dochterman, R. W. (1974). Centrifugal-vortex pump, United States Patent 3,936,240.

[12] Mihalić, T., Guzović, Z., \& Sviderek, S. (2011). Improving centrifugal pump by adding vortex rotor. Journal of Energy Technology, 11-20.

[13] Tesla, N. (1911). Dr. Tesla Talks of Gas Turbines. Motor World.

[14] Taylor, G. I. (1923). Stability of a Viscous Liquid contained between Two Rotating Cylinders. Phil. Trans. Royal Society, A223, 289-343. https://doi.org/10.1098/rsta.1923.0008

[15] Metcalfe, R. W., Hussain, F., Menon,S., \& Hayakawa, M. (1985). Coherent structures in a turbulent mixing layer: a comparison between numerical simulations and experiments. Turbulent Shear Flows, 5, 110-123. https://doi.org/10.1007/978-3-642-71435-1_11

\section{Contact information:}

Tihomir MIHALIĆ, doc. dr. Sc.

(Corresponding author)

University of Applied Science Karlovac,

I. Meštrovića 10, 47000 Karlovac, Croatia

E-mail: tihomir.mihalic@vuka.hr

Živko KONDIĆ, prof. dr. sc.

University North,

Ulica 104 brigade br.3, 42000 Varaždin, Croatia

E-mail: zivko.kondic@unin.hr

Srđan MEDIĆ, doc. dr. sc.

University of Applied Science Karlovac,

I. Meštrovića 10, 47000 Karlovac, Croatia

E-mail: smedic@vuka.hr

Veljko KONDIĆ, mag. ing. mech.

University North,

Ulica 104 brigade br.3, 42000 Varaždin, Croatia

E-mail: veljko.kondic@unin.hr 